\title{
Maturidade sexual morfológica de Aegla platensis (Crustacea, Decapoda, Anomura) no Lajeado Bonito, norte do estado do Rio Grande do Sul, Brasil
}

\author{
Davi de Oliveira \& Sandro Santos
}

Programa de Pós-Graduação em Biodiversidade Animal, Laboratório de Carcinologia, Departamento de Biologia, Centro de Ciências Naturais e Exatas, Universidade Federal de Santa Maria, Av. Roraima, 1000, 97105-900 Santa Maria, RS, Brasil. (ssantos@smail.ufsm.br)

\begin{abstract}
Morphological sexual maturity of Aegla platensis (Crustacea, Decapoda, Anomura) in the Lajeado Bonito, north of the state of Rio Grande do Sul, Brazil. The size at the onset of morphological sexual maturity (SOM) of the anomuran crab Aegla platensis Schmitt, 1942 was estimated through the changes in the ratios of corporal dimensions of the animals. For this, aeglids were monthly collected, from July 2007 to June 2008 in Lajeado Bonito $\left(27^{\circ} 25^{\prime} 27^{\prime \prime} \mathrm{S}, 53^{\circ} 24^{\prime} 39^{\prime \prime} \mathrm{W}\right)$, a first order tributary of the rio da Várzea, in Frederico Westphalen municipality, state of Rio Grande do Sul, Brazil. Samples of 437 males and 368 females were used, with cephalothorax length (CC) ranging from $6.00 \mathrm{~mm}$ to $31.75 \mathrm{~mm}$ in males and from $6.08 \mathrm{~mm}$ to $27.92 \mathrm{~mm}$ in females. The following corporal dimensions of collected individuals were measured: cephalothoracic length, abdomen width (LA), length of the right propodus cheliped (CPD), length of the left propodus cheliped (CPE). After the register of these measures, the crabs were returned to the stream, to the same places where they were collected. Estimation of the size at the onset of morphological maturity was performed using the Mature 2 software. The most appropriated relations for this analysis in males were CPD x CC (SOM: CC $=18.2 \mathrm{~mm}$ ) and CPE x CC (SOM: $\mathrm{CC}=20.1 \mathrm{~mm})$ and for females LA x CC (SOM: CC=16.5 mm).
\end{abstract}

KEYWORDS. Allometry, puberal molting, intraspecific variability.

RESUMO. O tamanho da primeira maturação sexual (TPM) em Aegla platensis Schmitt, 1942 foi estimado através das mudanças nas proporções de dimensões corporais dos animais. Para isso, foram realizadas coletas mensais, de julho de 2007 a junho de 2008 no Lajeado Bonito ( $\left.27^{\circ} 25^{\prime} 27^{\prime \prime} \mathrm{S}, 53^{\circ} 24^{\prime} 39^{\prime \prime} \mathrm{W}\right)$, um tributário de primeira ordem do Rio da Várzea, município de Frederico Westphalen, Rio Grande do Sul. Foram utilizados 437 machos com comprimento de cefalotórax (CC) variando de $6,00 \mathrm{~mm}$ a $31,75 \mathrm{~mm}$ e 368 fêmeas, com tamanhos entre $6,08 \mathrm{~mm}$ e $27,92 \mathrm{~mm}$ de CC. As seguintes dimensões corporais foram mensuradas em todos os indivíduos coletados: comprimento do cefalotórax (CC), largura do abdome (LA), comprimento do própodo do quelípodo direito (CPD) e comprimento do própodo do quelípodo esquerdo (CPE). Após o registro dessas medidas, os animais foram devolvidos ao mesmo local de captura. As análises de maturidade sexual morfológica foram realizadas com auxílio do software Mature 2, nas quais foram utilizadas as medidas de CC, considerada como variável independente e relacionada com as demais dimensões. As relações que melhor se ajustaram para estas análises, em machos, foram CPD x CC (TPM: CC=18,2 mm) e CPE x CC (TPM: CC=20,1 mm) e LA x CC (TPM: CC=16,5 mm) nas fêmeas.

PALAVRAS-CHAVE. Alometria, muda puberal, variabilidade intraespecífica.

O estudo de características morfométricas, durante a ontogênese, tem permitido inferir o tamanho em que determinada espécie atinge a maturidade sexual morfológica, eliminando o trabalho exaustivo de cultivo em laboratório (MASUNARI \& DisSENHA, 2005). A estimativa do tamanho mínimo dos indivíduos sexualmente maduros, em crustáceos, é facilitada, entre outros fatores, pelo tegumento rígido e de fácil mensuração e a presença de uma muda puberal definindo distintamente uma fase pré-puberal da puberal, acompanhada de mudanças bruscas nas proporções das dimensões do corpo (HARTNOLl, 1978). Essas alterações bruscas podem ser detectadas num gráfico de dispersão dos pontos empíricos de duas dimensões do corpo (sendo uma delas a de referência), através do ponto de inflexão entre dois ou mais possíveis modelos de crescimento (Masunari \& Dissenha, 2005). Em crustáceos, a relação alométrica entre o tamanho do corpo e vários órgãos tem sido utilizada para estimar o tamanho na maturidade, assumindo que os caracteres sexuais secundários aparecem e se desenvolvem de maneira diferente entre as fases imatura e madura (HAEFNER, 1990).

Os anomuros do gênero Aegla Leach, 1820 são animais de hábitos bentônicos, encontrados em arroios e rios de correnteza, ocultos sob pedras e detritos vegetais. Destacam-se como elos importantes nas cadeias alimentares nos ambientes límnicos, pois, além de serem predadores de larvas aquáticas (MAGNI \& PY-DANIEL, 1989; BuENO \& BONDBuckup, 2004; CASTRO-SOUZA \& BOND-BuCKuP, 2004; SANTOS et al., 2008), constituem importante fonte alimentar para peixes, anfíbios, aves e mamíferos (ARENAS, 1974; CASSINI et al., 2009).

Considerando-se que a análise da maturidade sexual está associada à biologia reprodutiva dos organismos, percebe-se que, embora alguns aspectos da reprodução dos eglídeos venham sendo estudados ultimamente (TUDGE \& Scheltinga, 2002; Sokolowicz et al., 2006; Sokolowicz et al., 2007), são poucos os trabalhos onde a maturidade é investigada de forma mais detalhada.

BuEno \& Bond-Buckup (2000) estimaram a maturidade sexual de Aegla platensis Schmitt, 1942, provenientes do Arroio do Mineiro, tributário do rio dos Sinos (Sistema Jacuí/Guaíba), município de Taquara, Rio Grande do Sul, com base no tamanho médio onde 50\% das fêmeas estavam ovígeras. CoLPO et al. (2005), utilizando análises de crescimento alométrico, determinaram o tamanho da maturidade sexual morfológica em Aegla longirostri Bond-Buckup \& Buckup, 1994, no rio Ibicuí-Mirim em Itaara, RS. VIAU et al. (2006) analisaram a maturidade sexual de Aegla uruguayana Schmitt, 1942 com base em dados morfológicos, histológicos e funcionais.

Bueno \& Shimizu (2008) estudaram o período reprodutivo, fecundidade e maturidade sexual funcional em 
fêmeas de Aegla franca Schmitt, 1942 no município de Claraval, Minas Gerais. Em seguida estes mesmos autores analisaram o crescimento alométrico e a maturidade sexual de machos e fêmeas de A. franca (BuENo \& SHIMIZu, 2009).

Considerando que até o momento são poucos os trabalhos que contribuem com informações sobre o tamanho de início da maturidade sexual em eglídeos, o presente estudo propõe-se a estimar, com base em dados morfológicos, este aspecto da biologia de A. platensis, provenientes de uma sub-bacia do alto rio Uruguai. Com estes dados será possível, também, avaliar se este parâmetro biológico varia entre populações de diferentes bacias hidrográficas.

\section{MATERIAL E MÉTODOS}

A área de estudo localiza-se no município de Frederico Westphalen, região norte do estado do Rio Grande do Sul (27²5'27'S, 5324'39'W). O local estudado é um trecho do Lajeado Bonito, tributário de primeira ordem do rio da Várzea, o qual faz parte da bacia do Rio Uruguai. No local onde as coletas foram realizadas, a altitude é de $470 \mathrm{~m}$ acima do nível do mar. A nascente do lajeado encontra-se próxima à sede do distrito de Oswaldo Cruz e as áreas no entorno são ocupadas por atividades agropecuárias, embora as margens do arroio sejam protegidas por mata ciliar. O leito do arroio é formado, em parte, por um afloramento rochoso (lajeado), por rochas soltas de variados tamanhos e sedimento arenoso.

Coleta dos animais. Os exemplares de A. platensis foram coletados mensalmente de julho de 2007 a junho de 2008 em um trecho de $150 \mathrm{~m}$ de extensão ao longo do Lajeado Bonito. Foram capturados com o auxílio de armadilhas confeccionadas com garrafa pet contendo isca de fígado bovino como atrativo aos animais. As referidas armadilhas $(n=16)$ foram colocadas antes do anoitecer, em locais propícios para a presença de eglídeos, como a presença de substrato para refúgio, e revisadas na manhã do dia seguinte. Para captura dos animais, também foi utilizado um puçá de $30 \mathrm{~cm}$ x $50 \mathrm{~cm}$ de boca com profundidade de $60 \mathrm{~cm}$ e malha com abertura de $1,0 \mathrm{~mm}$, sendo esse posicionado de maneira que os animais resultantes do revolvimento do substrato fossem carregados pela correnteza para dentro do mesmo.

Os animais capturados foram identificados quanto ao sexo e a presença de fêmeas ovígeras foi registrada. A sexagem foi realizada com base em caracteres morfológicos, como presença de pleópodos nas fêmeas e ausência desses nos machos. As medidas tomadas com auxílio de um paquímetro digital com precisão de $0,01 \mathrm{~mm}$ foram: comprimento do cefalotórax (CC, da parte distal do rostro até o bordo posterior da carapaça), largura do cefalotórax (LC, distância entre as margens epibranquiais esquerda e direita), comprimento dos própodos dos quelípodos direito (CPD) e esquerdo (CPE) e largura do abdome (LA, largura do segundo somito abdominal). Indivíduos com tamanho abaixo de $8 \mathrm{~mm}$ de CC foram medidos em laboratório, também com um paquímetro, mas sob um estereomicroscópio, distinguindo-se os sexos pela presença de poro genital na coxa do terceiro par de pereiópodos nas fêmeas e a ausência desses nos machos. Após a coleta dos dados, os animais foram devolvidos ao mesmo local de captura. Uma amostra de exemplares está depositada na coleção do Laboratório de Carcinologia da Universidade Federal de Santa Maria.

Análise dos dados. A análise de maturidade sexual morfológica foi realizada com base na relação alométrica entre o tamanho do corpo (CC) e as demais dimensões do animal, assumindo que os caracteres sexuais secundários aparecem e crescem em taxas diferentes entre a fase imatura e madura. Considerando-se essas diferenças de crescimento, é possível determinar o ponto de inflexão na tendência apresentada pelos pontos bivariados, onde há a separação da fase juvenil da adulta. Desta maneira as dimensões que apresentam taxas de crescimento estatisticamente distintas, entre as fases de vida, são aquelas indicadas para a determinação da maturidade sexual em crustáceos.

Os dados do presente estudo, quando plotados em gráficos, apontam para retas ajustadas aos pontos referentes a juvenis e adultos que se interceptam em pontos nítidos, sem que estas se sobreponham, justificando a utilização do software Mature 2, de acordo com SOMERTON (1980). Para fêmeas, foi utilizadaa relação LAx CC, LCx CC, CPDx CC, CPE xCCepara machos, CPD xCC; CPExCC; LAxCCeLCxCC.

Para efeito de comparações, após o cálculo dos tamanhos de maturidade realizado no programa Mature 2, os dados de juvenis e adultos foram ajustados para equações alométricas $\mathrm{Y}=\mathrm{a} \mathrm{X}^{\mathrm{b}}(\mathrm{Y}=$ variáveis dependentes; $\mathrm{X}=$ variável independente: $\mathrm{CC} ; \mathrm{b}=$ coeficiente de crescimento alométrico e $\mathrm{a}=$ intercepto de $\mathrm{Y})$ e posteriormente linearizados $(\ln \mathrm{Y}=$ lna $+b X)$. As análises de regressão foram desenvolvidas no programa BioEstat 5.0 (AYREs et al., 2007). Para testar se o coeficiente de crescimento alométrico diferia do valor 1 (crescimento isométrico), aos dados obtidos foi aplicado o test $t$ de Student $\left(\mathrm{H}_{0}: \mathrm{b}=1\right)$ (SoKal \& RohlF, 1979).

Os parâmetros das regressões lineares, obtidos para juvenis e adultos de cada sexo, foram comparados com base em uma análise de covariância (ANCOVA) para um nível de significância $(\alpha)$ de 0,05 (SoKAL \& RoHLF, 1979), utilizandose o programa BioEstat (AYres et al., 2007).

\section{RESULTADOS}

No total foram amostrados 957 animais, dos quais foram utilizados 437 machos e 368 fêmeas que possuíam os apêndices do corpo em condições de serem mensurados. O comprimento do cefalotórax (CC) variou de 6,00 mm a 31,75 mm em machos e de 6,08 mm a 27,92 mm em fêmeas.

Nos machos, as relações entre as dimensões corpóreas que apresentaram indicações de maturidade sexual morfológica foram CPE x CC e CPD x CC (Tab. I), sendo possível ajustá-las em duas retas com o auxílio do software Mature 2 (Figs 1,2). O referido software estimou a maturidade sexual morfológica de machos em $18,2 \mathrm{~mm}$ para CPD x CCe 20,1 mm para CPE x CC. Há diferença significativa entre o crescimento destes dois quelípodos (teste $t \mathrm{p}<0,05$ ), caracterizando uma heteroquelia nos machos adultos de $A$. platensis. Para efeitos práticos, sugere-se a adoção da média dos valores estimados, $19,15 \mathrm{~mm}$ de CC, como o tamanho médio na primeira maturação de machos de A. platensis no Lajeado Bonito.

Para a análise de fêmeas, a medida que melhor se ajustou para o cálculo de maturidade foi de LA x CC (Fig. 3). A estimativa de tamanho da maturidade sexual feita pelo software Mature 2, com base na largura do abdome, foi de $16,5 \mathrm{~mm}$ de CC. A menor fêmea ovígera apresentou $16,7 \mathrm{~mm}$ de CC e a maior $24,87 \mathrm{~mm}$.

Há diferenças significativas entre os parâmetros das equações lineares ajustadas para juvenis e adultos machos, tanto se considerando as dimensões CPD quanto CPE, e entre as fêmeas, com base na largura do abdome, as diferenças também são significativas (Tab. II). 
Tabela I. Resultados da análise alométrica realizada com Aegla platensis Schmitt, 1942 no Lajeado Bonito, Frederico Westphalen, Rio Grande do Sul (CC, comprimento do cefalotórax; CPD, comprimento do própodo direito; CPE, comprimento do própodo esquerdo; FA, fêmeas adultas; FJ, fêmeas juvenis; LA, largura do abdome; MA, machos adultos; MJ, machos juvenis; n, número de exemplares).

\begin{tabular}{|c|c|c|c|c|}
\hline Variável & Sexo & $\mathrm{N}$ & Equação Linear; $\mathrm{r}^{2}(\mathrm{Y}=\mathrm{a}+\mathrm{bX})$ & $\begin{array}{l}\text { Teste t de Student para a alometria } \\
\qquad(\mathrm{Ho}: \mathrm{b}=1) ; \text { alometria }\end{array}$ \\
\hline \multirow[t]{2}{*}{$\overline{\mathrm{CPD}}$} & MJ & 271 & $\mathrm{CPD}=1,071 \mathrm{CC}-0,930 ; 0,97$ & $\mathrm{t}=89,965, \mathrm{p}<0,001 ;+$ \\
\hline & MA & 161 & $\mathrm{CPD}=1,721 \mathrm{CC}-2,872 ; 0,91$ & $\mathrm{t}=39,426, \mathrm{p}<0,001 ;+$ \\
\hline \multirow[t]{2}{*}{ CPE } & MJ & 302 & $\mathrm{CPE}=1,162 \mathrm{CC}-1,112 ; 0,96$ & $\mathrm{t}=88,850, \mathrm{p}<0,001 ;+$ \\
\hline & MA & 155 & $\mathrm{CPE}=1,836 \mathrm{CC}-3,138 ; 0,87$ & $\mathrm{t}=31,947, \mathrm{p}<0,001 ;+$ \\
\hline \multirow[t]{2}{*}{ LA } & FJ & 184 & $\mathrm{LA}=1,112 \mathrm{CC}-0,640 ; 0,98$ & $\mathrm{t}=105,39, \mathrm{p}<0,001 ;+$ \\
\hline & FA & 187 & $\mathrm{LA}=1,268 \mathrm{CC}-1,078 ; 0,96$ & $\mathrm{t}=64,958, \mathrm{p}<0,001 ;+$ \\
\hline
\end{tabular}

\section{DISCUSSÃO}

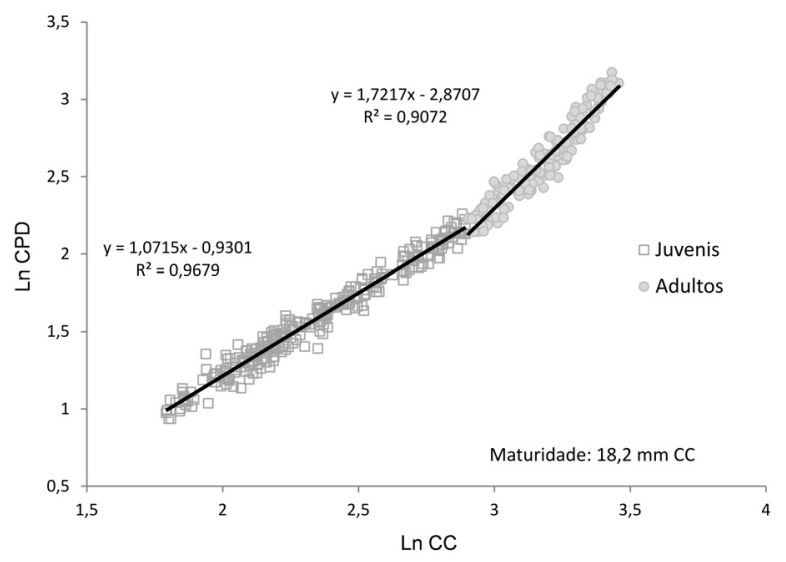

Figura 1. Aegla platensis Schmitt, 1942. Diagramas de dispersão de pontos das relações morfométricas entre o comprimento do própodo do quelípodo direito e o comprimento do cefalotórax de machos.

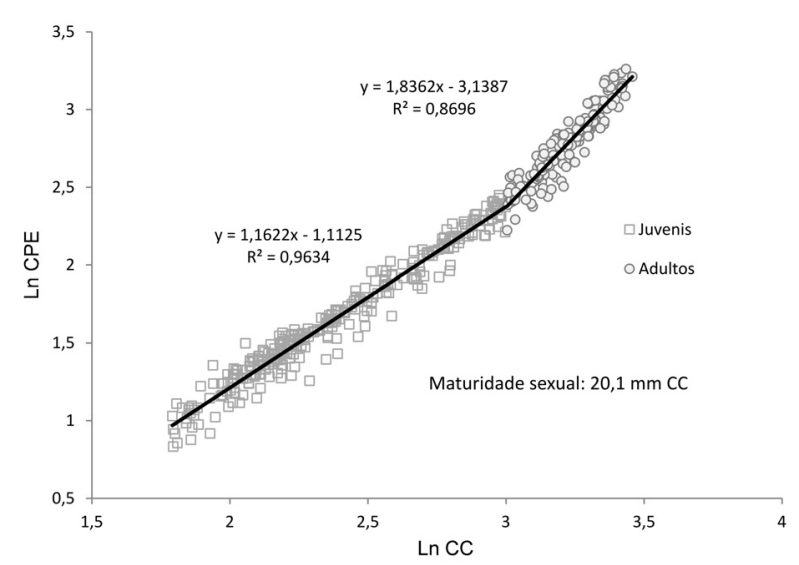

Figura 2. Aegla platensis Schmitt, 1942. Diagramas de dispersão de pontos das relações morfométricas entre o comprimento do própodo do quelípodo esquerdo e o comprimento do cefalotórax de machos.

Tabela II. Comparação dos parâmetros das regressões lineares entre juvenis e adultos de Aegla platensis Schmitt, 1942 (CPD, comprimento do própodo direito; CPE, comprimento do própodo esquerdo; LA, largura do abdome).

\begin{tabular}{lcl}
\hline Sexo & Dimensão & Coeficiente de regressão (b) \\
\hline Machos & CPD & $\mathrm{F}=268,389 ; \mathrm{v}=427 ; \mathrm{p}=0,0000$ \\
& CPE & $\mathrm{F}=149,103 ; \mathrm{v}=453 ; \mathrm{p}=0,0000$ \\
Fêmeas & LA & $\mathrm{F}=43,120 ; \mathrm{v}=367 ; \mathrm{p}=0,0000$ \\
\hline
\end{tabular}

Atualmente ainda são poucos os trabalhos que analisam a maturidade sexual morfológica com base em características morfométricas em espécies de anomuros do gênero Aegla. A maioria dos estudos realizados, com base nestas características, é com crustáceos da Infraordem Brachyura. Considerando-se que esta técnica pode evitar que os animais estudados sejam sacrificados, e que onde houve comparações com outros métodos os resultados apresentam pouca variação (SANTOS \& Negreiros-Fransozo, 1995), esta técnica deveria receber uma atenção preferencial.

Embora em todas as relações estudadas o crescimento seja registrado como alométrico positivo, com base nas análises de covariância verifica-se que há mudança no modelo de crescimento, representada pelas diferenças nas equações lineares entre estas categorias etárias.

ColPo et al. (2005) estimaram a maturidade sexual em Aegla longirostri e os valores obtidos foram de 13,7 mm de CC para machos e 8,6 mm para fêmeas. Nas fêmeas, o CC no momento em que $50 \%$ delas estavam maduras, foi de 10,7 $\mathrm{mm}$. Assim como no presente estudo, foi verificado que as relações CC x comprimento dos quelípodos são as que apresentaram melhores respostas na avaliação de maturidade em machos e em fêmeas a melhor relação foi LA x CC.

Viau et al. (2006), ao estudarem indivíduos de $A$. uruguayana provenientes do rio Areco, na Argentina, estimaram a maturidade sexual morfológica de fêmeas em 11,5 mm. Porém, esse valor é inferior ao obtido pela observação de gônadas, estimado entre 15 e 17 mm de CC. Neste mesmo estudo, a menor fêmea ovígera apresentou 15,6 mm de CC. Para machos o valor encontrado foi de 15,4 $\mathrm{mm}$, também inferior ao observado em análises de gônadas, $18,7 \mathrm{~mm}$ de CC. Esses autores também verificaram que a relação comprimento dos quelípodos x CC foi a que proporcionou a mais clara distinção entre jovens e adultos, indicando o ponto de maturação em machos.

Considerando-se o tamanho máximo que os indivíduos de cada espécie podem atingir, e calculando-se a maturidade em função da porcentagem daquele tamanho, observa-se que a maturidade, em eglídeos, é atingida entre aproximadamente 40 e $70 \%$ do tamanho máximo. $\mathrm{O}$ comprimento na maturidade sexual, estimado no presente estudo, é superior ao de outros estudos realizados até o momento, exceto quando se compara às fêmeas de $A$. franca estudadas por BuENo \& SHIMIZU (2009). Neste estudo a maturidade sexual foi atingida com $59,95 \%$ do tamanho máximo atingido pelas fêmeas daquela população (Tab. III).

BuENO \& BOND-BuCKUP (2000), em análises feitas com A. platensis provenientes da bacia hidrográfica do rio Jacuí/ Guaíba, estimaram o tamanho da primeira maturação em fêmeas entre 14,40e 15,60mm deCC, porém, esta estimativa não 
Tabela III. Tamanho da primeira maturação de diferentes espécies de Aegla Leach, 1820, considerando-se a proporção em relação ao tamanho máximo atingido pelos animais de cada espécie, para as quais foram utilizados dados morfométricos para a estimativa de maturidade (F, fêmea; M, macho)

\begin{tabular}{|c|c|c|c|c|c|c|c|}
\hline \multirow[t]{2}{*}{ Espécie } & \multicolumn{2}{|c|}{$\begin{array}{l}\text { Tamanho } \\
\text { máximo }(\mathrm{mm})\end{array}$} & \multicolumn{2}{|c|}{$\begin{array}{c}\text { Tamanho de } \\
\text { maturação }(\mathrm{mm})\end{array}$} & \multicolumn{2}{|c|}{$\begin{array}{l}\text { Proporção do tamanho } \\
\text { máximo na maturação }\end{array}$} & \multirow[t]{2}{*}{ Referências } \\
\hline & $\mathrm{M}$ & $\mathrm{F}$ & $\mathrm{M}$ & $\mathrm{F}$ & $\mathrm{M}$ & $\mathrm{F}$ & \\
\hline Aegla longirostri & 23,80 & 18,90 & 13,70 & 10,70 & 57,56 & 56,61 & Colpo et al. (2005) \\
\hline A. uruguayana & 34,80 & 29,80 & 15,40 & 11,50 & 44,25 & 38,59 & VIAU et al. (2006) \\
\hline A. franca & 25,62 & 19,70 & 14,40 & 11,81 & 56,21 & 59,95 & Bueno \& Shimizu (2009) \\
\hline A. platensis & 31,75 & 27,92 & 19,15 & 16,50 & 60,31 & 59,10 & Presente estudo \\
\hline
\end{tabular}

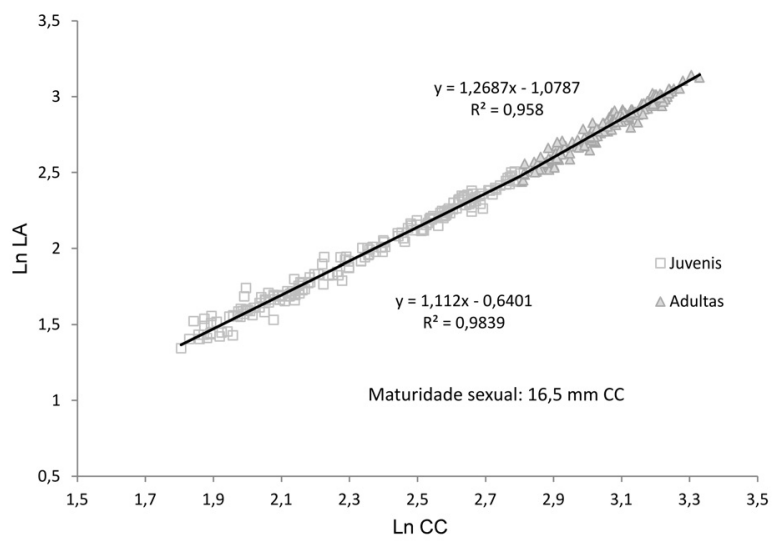

Figura 3. Aegla platensis Schmitt, 1942. Diagramas de dispersão de pontos das relações morfométricas entre a largura do abdome e o comprimento do cefalotórax em fêmeas.

foi realizada em função de dados morfométricos, por esta razão não se calculou a porcentagem em relação ao tamanho máximo dos animais. Naquele estudo a menor e a maior fêmea ovígera apresentavam 9,87 e 17,72 $\mathrm{mm}$ de CC, respectivamente. O tamanho da menor e da maior fêmea ovígera, registrado pelos referidos autores, é inferior ao registrado no presente estudo (16,7 mme 24,87 mm, respectivamente-bacia do rio Uruguai). Estas diferenças de tamanho indicam que há variações significativas no desenvolvimento de populações de uma mesma espécie habitando diferentes bacias hidrográficas. Estas diferenças, provavelmente, são decorrentes de condições ambientais inerentes de cada bacia hidrográfica.

O tamanho médio em que os indivíduos de cada população atingem a maturidade, em função do tamanho máximo, também pode ser correlacionado às condições ambientais onde as populações vivem. Estas diferenças também podem ser atribuídas a características próprias de cada espécie, porém estas hipóteses precisariam ser testadas com base em estudos com mais espécies e populações de diferentes localidades.

Agradecimentos. Ao Dr. Adilson Fransozo e a Dra. Karine Delevati Colpo, pelas sugestões e revisão do manuscrito sob a forma de dissertação de mestrado do primeiro autor (DO). Ao CNPq pela bolsa de pesquisa do segundo autor (SS).

\section{REFERÊNCIAS BIBLIOGRÁFICAS}

ArENAS, J. 1974. La cordillera de la costa como refugio de la fauna dulcícola preglacial. Archivos de Biologia y Medicina Experimentales 10:1-40.

Ayres, M.; Ayres Jr., M.; Ayres, D. L. \& SAntos, A. S. 2007. Bioestat 5.0: Aplicacõoes estatísticas nas áreas das Ciências Biológicas e Médicas. Belém, Sociedade Civil Mamirauá. 364p.

Bueno, A. A. P. \& Bond-Buckup, G. 2000. Dinâmica populacional de Aegla platensis Schmitt (Crustacea, Decapoda, Aeglidae). Revista Brasileira de Zoologia 17(1):43-49.
2004. Natural diet of Aegla platensis Schmitt and Aegla ligulata Bond-Buckup \& Buckup (Crustacea, Decapoda, Aeglidae) from Brazil. Acta Limnológica Brasileira 16(2):115-127.

Bueno, S. L. S. \& Shimizu, R. M. 2008. Reproductive biology and functional maturity in females of Aegla franca (Decapoda: Anomura: Aeglidae). Journal of Crustacean Biology 28(4):652-662.

2009. Allometric growth, sexual maturity, and adult male chelae dimorphism in Aegla franca (Decapoda, Anomura: Aeglidae). Journal of Crustacean Biology 29(3):317-328.

Cassini, M. H.; Fasola, L.; ChehÉBar, C. \&. MacDonald, D. W. 2009. Scale-dependent analysis of an otter-crustacean system in Argentinean Patagonia. Naturwissenschaften 96:593-599.

Castro-Souza, T. \& Bond-Buckup, G. 2004. O nicho trófico de duas espécies simpátricas de Aegla no tributário da bacia hidrográfica do Rio Pelotas, Rio Grande do Sul, Brasil. Revista Brasileira de Zoologia 21(4):805-813.

Colpo, K. D.; Ribeiro, L. D. \& Santos, S. 2005. Population biology of the freshwater Anomura Aegla longirostri (Aeglidae) from South Brazilian streams. Journal of Crustacean Biology 25(3):495-499.

HAEFNER JR., P. A. 1990. Morphometry and size at maturity of Callinectes ornatus (Brachyura, Portunidae) in Bermuda. Bulletin of Marine Science 46(2):264-286.

Hartnoll, R. G. 1978. The determination of relative growth in Crustacea. Crustaceana 34(3):281-289.

Magni, S. T. \& Py-Daniel, V. 1989. Aegla platensis Schmitt, 1942 (Decapoda, Anomura) um predador de imaturos de Simuliidae (Diptera, Culicomorpha). Revista de Saúde Pública 23(Supl. 3):258-259.

Masunari, S. \& Dissenha, N. 2005. Alometria no crescimento de Uca mordax (Smith) (Crustacea, Decapoda, Ocypodidae) na Baía de Guaratuba, Paraná, Brasil. Revista Brasileira de Zoologia 22(4):984-990.

Santos, S.; Cardoso, R. C. F.; Ayres-Peres, L. \& Sokolowicz, C. C. 2008. Natural diet of the freshwater anomuran Aegla longirostri (Crustacea, Anomura, Aeglidae). Journal of Natural History 42:1027-1037.

Santos, S. \& Negreiros-Fransozo, M. L. 1995. Morphometric relationship and maturation in the Portunus spinimanus Latreille, 1819 (Crustacea, Decapoda, Portunidae). Revista Brasileira de Biologia 55(4):545-553.

Sokal, R. R. \& Rohlf, F. J. 1979. Biometria. Principios y métodos estadísticos en la investigación biológica. Madrid, H. Blumes Ediciones. 832p.

Sokolowicz, C. C.; Bond-Buckup, G. \& Buckup, L. 2006. Dynamics of gonadal development of Aegla platensis Schmitt (Decapoda Anomura, Aeglidae). Revista Brasileira de Zoologia 23(4): 1153-1158

Sokolowicz, C. C.; López-Greco, L. S.; GonçAlves, R. \& Bond-Buckup, G. 2007. The gonads of Aegla platensis Schmitt (Decapoda, Anomura, Aeglidae): a macroscopic and histological perspective. Acta Zoologica 88:71-79.

Somerton, D. A. 1980. A computer technique for estimating the size of sexual maturity in crabs. Canadian Journal of Fishery and Aquatic Sciences 37:1488-1494.

Tudge, C. C. \& Scheltinga, D. M. 2002. Spermatozoal morphology of the freshwater anomuran, Aegla longirostri Bond-Buckup \& Buckup, 1994 (Crustacea: Decapoda: Aeglidae) from South America. Proceedings of the Biological Society of Washington 115(1): 118-128.

Viau, V. E.; López Greco, L. S.; Bond-Buckup, G. \& Rodríguez, E. M. 2006. Size at onset of sexual maturity in anomuran crab, Aegla uruguayana (Aeglidae). Acta Zoologica 87:253-264.

Recebido em outubro de 2010. Aceito em maio de 2011. ISSN 0073-4721

Artigo disponível em: www.scielo.br/isz 